\title{
Griffiths singularities and magnetoresistive manganites
}

\author{
M. B. Salamon* \\ Department of Physics, University of Illinois, \\ 1110 W. Green Street Urbana, IL 61801 \\ S. H. Chun $^{\dagger}$ \\ Department of Physics, Sejong University, Seoul 143-747, Korea
}

(Dated: October 30, 2018)

\begin{abstract}
The large, so-called colossal, magnetoresistivity of doped manganese oxides based on $\mathrm{LaMnO}_{3}$ has attracted considerable attention, but only one unusual feature of the ferromagnetic transition in these compounds. We examine in this paper the progression of magnetic and thermodynamic behavior as the transition temperature is made to vary from $360 \mathrm{~K}$ to $218 \mathrm{~K}$ by changing the divalent dopant. Single crystals of $\mathrm{La}_{0.7} \mathrm{Sr}_{0.3} \mathrm{MnO}_{3}$, as is well known, show modest magnetoresistivity and conventional critical behavior. $\mathrm{La}_{0.7} \mathrm{~Pb}_{0.3} \mathrm{MnO}_{3}$, and to an even greater extent, $\mathrm{La}_{0.7} \mathrm{Ca}_{0.3} \mathrm{MnO}_{3}$, have unusual magnetic properties extending more than $100 \mathrm{~K}$ above the transition. We treat the properties of the latter samples in the context of a Griffiths phase in which the transition temperature is depressed from its maximum value $T_{G}$ by random bond-angle bending.
\end{abstract}

*Electronic address: salamon@uiuc.edu

${ }^{\dagger}$ Electronic address: schun@sejong.ac.kr 


\section{INTRODUCTION}

The properties of $\mathrm{AMnO}_{3}$, where $\mathrm{A}$ is a mixture of trivalent lanthanides and divalent ions, have intrigued researchers for decades.[1] The parent compound, $\mathrm{LaMnO}_{3}$, crystallizes in a slightly distorted perovskite structure and is an antiferromagnetic insulator with a Neèl temperature $T_{N} \approx 130 \mathrm{~K}$. When concentration of divalent atoms (Ca, Sr, Ba, Pb..) substituted for La (A-site substitution) exceeds $\approx 1 / 8$, the low temperature phase is ferromagnetic and metallic. The Curie temperature depends strongly on the concentration and ionic size of the substituent [2] and, perhaps most significantly, on the ionic-size variance of A-site atoms. [3] The highest Curie temperature, $T_{C} \approx 360 \mathrm{~K}$, is achieved with $\mathrm{Sr}$ doping at a concentration close to $3 / 8$; that is, for $\mathrm{La}_{5 / 8} \mathrm{Sr}_{3 / 8} \mathrm{MnO}_{3}$. At this concentration, the material is metallic in both paramagnetic $(T \geq 360 \mathrm{~K})$ and ferromagnetic phases, and the effect of magnetic fields on the electrical resistivity is not dramatic. The ferromagnetic/paramagnetic transition is entirely normal, by which we mean that the magnetization can be described by critical exponents very close to those expected for a three-dimensional Heisenberg ferromagnet. [4]

The conventional picture for this system is based on the double exchange mechanism proposed by Zener.[5] Each divalent substituent converts a $\mathrm{Mn}^{3+}$ ion to $\mathrm{Mn}^{4+}$, with the outermost $\left(e_{g}\right)$ electron on $\mathrm{Mn}^{3+}$ site resonating with a neighboring $\mathrm{Mn}^{4+}$ via the intervening oxygen atom. Because of strong Hund's-rule coupling, the double-exchange transfer is favored when neighboring core spins are aligned, leading to ferromagnetism. When the substitution level is sufficiently high, the holes doped into this system form a fully spin-polarized (half-metallic) band. As the $S=3 / 2$ core $\left(t_{2 g}\right)$ spins disorder with increasing temperature, the resistivity increases and, near the Curie temperature, exhibits substantial-though not dramatic-magnetoresistance. This picture describes $\mathrm{La}_{5 / 8} \mathrm{Sr}_{3 / 8} \mathrm{MnO}_{3}$ reasonably well. [6]

Changing the Sr content away from $\mathrm{La}_{5 / 8} \mathrm{Sr}_{3 / 8} \mathrm{MnO}_{3}$, substituting $\mathrm{Ca}$ or other divalent atoms for Sr at the same concentration and even substituting other lanthanides for La sharply decreases $T_{C}$ and dramatically changes the nature of the paramagnetic/ferromagnetic transition. The resistivity in the paramagnetic phase increases exponentially with decreasing temperature, peaks somewhat above $T_{C}$, and then decreases sharply in the ferromagnetic phase. The resistivity peak shifts to higher temperature with increasing field, giving rise

to the dramatic field dependent resistivity that has been termed colossal magnetoresistance (CMR). A calculation of the resistivity within the context of the double-exchange model 
[7] provided strong evidence that a localizing mechanism beyond that model was necessary to explain these large field- and temperature-dependent changes, and there is now strong theoretical [8] and experimental evidence [9] that polaron formation and accompanying selftrapping of electrons play essential roles. As the average ionic size of A-site atoms decreases toward that of La, the transition temperature decreases and the exponential increase in resistivity with temperature makes the drop to metallic resistivity at $T_{C}$ ever more dramatic. A powerful argument can be made that the smaller the A-site atom the greater is the distortion of the crystal from the cubic perovskite structure. The concurrent bending of the Mn-OMn bond angle inhibits the double-exchange resonance that drives ferromagnetic order and lowers $T_{C}$. [10] However, even if the average ionic size is kept constant (usually monitored by the so-called tolerance factor), the transition temperature drops as the variance in ionic size increases. [3] This suggests that local bond-angle bending is more important than the average and that disorder therefore plays a major role. Indeed, there is considerable evidence that metallic and polaronic regions coexist in the vicinity of the phase transition. The phase separation is dynamic, but much slower than is typical for critical fluctuations as can be seen in noise measurements [11, 12], muon spin relaxation [13], and the presence of strong diffusive peaks in neutron scattering. The case for phase separation, driven by the randomness inherent in the system, has been documented extensively in a recent review article by Dagotto. [14]

This paper explores the dramatic changes in thermodynamic behavior that accompany the better known changes in transport properties upon various substitutions away from $\mathrm{Sr}_{3 / 8}$. We will argue that bond disorder plays a key role and that the problem should be considered in the context of a Griffiths singularity. In his pioneering paper, Griffiths [15] considered a percolation-like problem in which each exchange bond in a system has value $J_{1}$ with probability $p$ and $J_{2}=0$ with probability $1-p$. For all $p<1$, Griffiths showed that the free energy, and thus the magnetization, is singular at the transition point $T_{C}(p)$, a consequence of the accumulation of clusters whose local transition temperatures exceed $T_{C}(p)$. Fisch [16] extended the argument to $0 \leq J_{2}<J_{1}$, demonstrating that the singularities persist. These results suggest, as emphasized by Dotsenko [17], that the essential contributions of local minima destroy the length-scaling picture of a random-fixed-point universality class. Bray and Moore [18] and Bray [19] extended the argument to any bond distribution that reduces the transition temperature from some "pure" value $T_{G}$ and proposed a distribution function 
for the inverse susceptibility tensor that captures the singularity proposed by Griffiths. Bray terms the temperature range $T_{C}(p) \leq T \leq T_{G}$ the Griffiths phase, where $p$ is now a measure of the bond distribution. The nature of the Griffiths singularity in the limit of small dilution has been treated in some detail in the quantum limit where $T_{C}(p) \rightarrow 0$ by Castro Neto and coworkers.[20, 21]

In this paper, which builds upon earlier work, [22] we demonstrate the progression of the magnetic and thermodynamic properties of doped $\mathrm{LaMnO}_{3}$ as the transition temperature is lowered from its maximum value. We then turn to an analysis of the low-field behavior of the magnetization based on the eigenvalues of the inverse susceptibility as proposed by Bray. In Section IV, we extend the analysis by introducing a bond distribution that changes with temperature and field as a consequence of the double-exchange mechanism and treat it using a cluster model. Section V concludes the paper with a discussion of the implications of this analysis for disordered double-exchange magnets.

\section{MAGNETIC AND THERMODYNAMIC PROPERTIES}

Three single-crystal samples were used in this study. Two samples, $\mathrm{La}_{0.7} \mathrm{Sr}_{0.3} \mathrm{MnO}_{3}$ (LSMO) and $\mathrm{La}_{0.7} \mathrm{Ca}_{0.3} \mathrm{MnO}_{3}$ (LCMO) were grown by optical floating-zone techniques by Okuda, et al.[23] A sample of $\mathrm{La}_{0.67}(\mathrm{~Pb}, \mathrm{Ca})_{0.33} \mathrm{MnO}_{3}$ (LPMO) was grown by flux methods as described elsewhere [24] and has a transition temperature midway between the extremes represented by the other samples. The LSMO and LPMO samples were cut into rectangular slabs with the long direction along the $a$ directions while the LPMO was used as grown, but had a similar orientation. The magnetization of each crystal was measured in a conventional Quantum Design MPMS system with the field along the longest axis of the sample. The data reported are corrected for demagnetization. Following the magnetic measurements, gold current and voltage pads were sputtered on the sample and leads were attached to the pads with silver paint. One end of the sample was varnished to a copper block while a strain gauge heater was attached to the opposite end. A pair of fine-wire thermocouples were connected to measure the temperature difference between the voltage contacts for thermopower measurements. The resistance and thermopower were measured sequentially at each field-temperature point in a Quantum Design PPMS instrument. Following the transport measurements, the samples were mechanically thinned, removing the gold contact pads, 


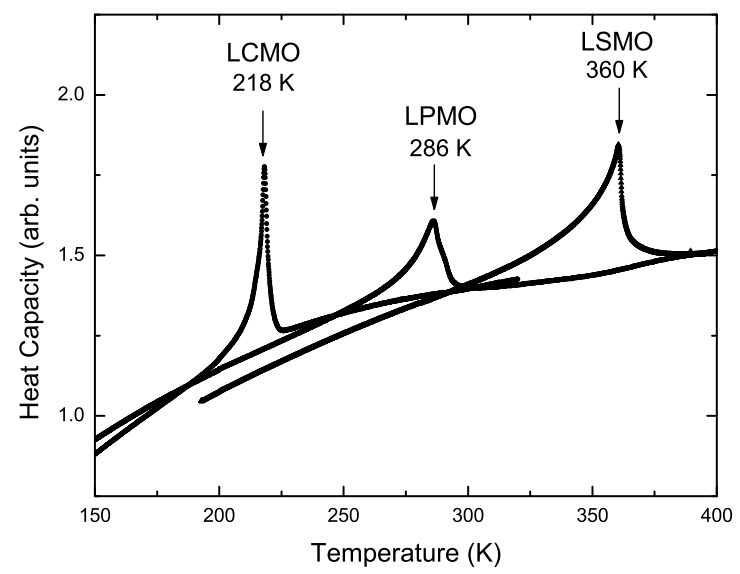

FIG. 1: Heat capacity in zero applied field for the three samples. The $\mathrm{La}_{0.7}(\mathrm{Ca}, \mathrm{Pb})_{0.3} \mathrm{MnO}_{3}$ sample was damaged upon thinning and shows a reduced heat capacity peak.

and mounted for ac calorimetry measurements. The small LPMO crystals were damaged in this process and the heat capacity data in field could not be obtained. Samples were placed in a crystat in which a magnetic field up to $7 \mathrm{~T}$ could be applied. Light from a stabilized quartz lamp was chopped mechanically to provide periodic heat pulses to the sample at the desired frequency. The proper operating point was located at the midpoint of the range where the ensuing temperature oscillations were inversely proportional to the frequency of the heat pulses. A thorough review of the ac method has been prepared by its inventor, Y. Kraftmakher. [25]

Figure 1 shows the ac heat capacity vs temperature for the three samples in zero applied field. Although the LPMO sample shows obvious signs of the damage that accompanied thinning, as noted above, the heat capacity exhibits a sharp peak at the temperatures indicated as $\mathrm{T}_{C}$ (heat capacity) in Table I. The heat capacity curve for LCMO is significantly narrower than for LSMO, a point we will address in more detail below. Despite its sharpness, there is no sign of hysteresis in the LCMO data. Similarly, the magnetization curves change significantly as the transition temperature is reduced. As had been reported previously [4], the magnetization for LSMO can be collapsed to a single curve using exponents that are similar to those expected for a Heisenberg ferromagnet. Our data behave similarly, as can be seen in Fig. 2 with the exponent values given in Table I. Here, $t=\left(T / T_{C}-1\right)$; the values of $T_{C}, \alpha$, and $\delta$ are those that best collapse the data above (upper curve) and below 


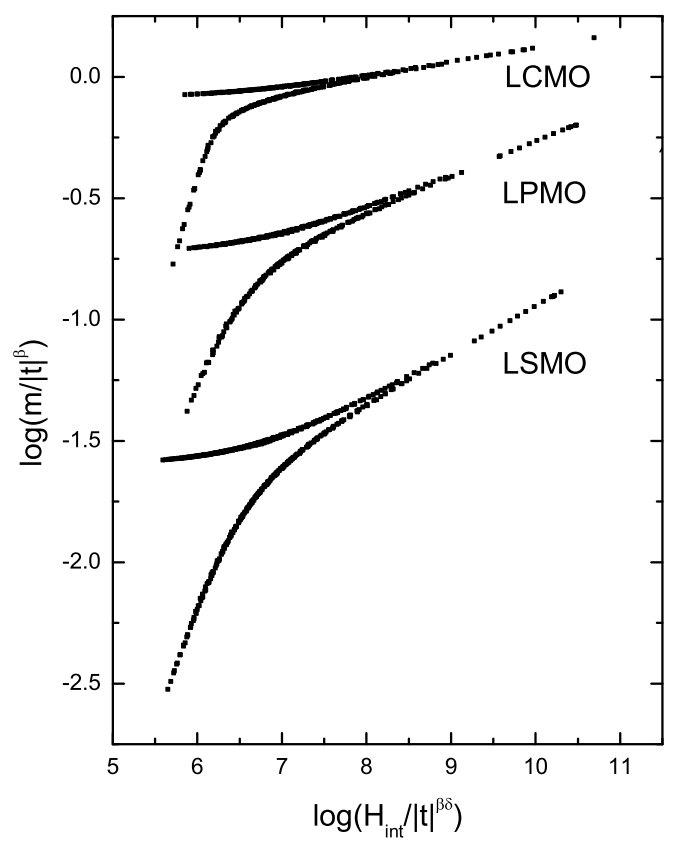

FIG. 2: Scaling curves for the three samples. The exponents $\beta$ and $\delta$ deviate strongly from Heisenberg-like values as $T_{C}$ is reduces. The temperatures in parentheses indicate the range of data used in the scaling curves.

\begin{tabular}{|l|l|l|l|l|}
\hline & $\mathrm{T}_{C}$ (heat capacity) & $\mathrm{T}_{C}($ scaling $)$ & $\beta$ & $\delta$ \\
\hline LCMO & $218 \mathrm{~K}$ & $216.2 \mathrm{~K}$ & 0.10 & 16.9 \\
LPMO & $286 \mathrm{~K}$ & $285.1 \mathrm{~K}$ & 0.24 & 7.1 \\
LSMO & $360 \mathrm{~K}$ & $359.1 \mathrm{~K}$ & 0.31 & 5.1 \\
\hline
\end{tabular}

TABLE I: Transition temperatures and critical exponents for samples studied.

(lower curve) $T_{C}$. The exponents are somewhat different from those reported by Ghosh et al. [4], but are also not far from the Heisenberg values $\beta=0.36$ and $\delta=4.8$. However, as the transition temperature decreases, the data can be collapsed only by using exponents that are far from those for any universality class.

The effects can be seen more directly by following the magnetization curves along the isotherms corresponding to the peaks in the zero-field heat capacity curves. The ratio of the measured magnetization at $T_{C}$ to the low-temperature saturation value is shown in Fig. 


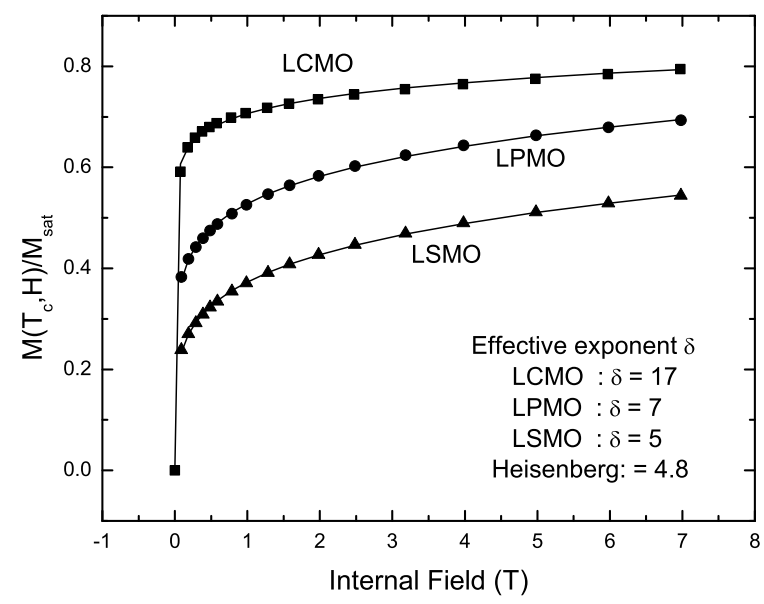

FIG. 3: Magnetization vs internal field along the critical isotherm. The exponent $\delta$ increases strongly as the transition temperature decreases.

3 for all three samples. The solid curves are fits the usual expression $M\left(H, T_{C}\right) \propto H^{1 / \delta}$ along the critical isotherm; the exponents agree with the scaling analysis. Note that the magnetization of LCMO rises to $60 \%$ of saturation in low fields, yet shows no signs of hysteresis or remanence. It is tempting to attribute this behavior to a first-order transition, but we will discuss it in the next section in terms of a Griffiths singularity.

While the LSMO data seem quite close to Heisenberg behavior, the low field susceptibility of that sample, as well as that of the others, is anomalous. Figure 4 shows the inverse susceptibility of the three samples normalized by the low temperature saturation magnetization $M(0)$ and plotted versus reduced temperature $T / T_{C}$. If these data followed a Curie-Weiss law, they would lie on a straight line given by

$$
H M(0) / M(T)=\frac{3 k_{B} T_{C}}{g \mu_{B}(S+1)}\left(\frac{T}{T_{C}}-1\right) .
$$

The dashed line is the slope expected for $T_{C}=360 \mathrm{~K}$ and $S=1.85$, namely the values for LSMO. The actual slope of the LSMO data corresponds to a spin $S \approx 3.5$ while that for LCMO requires $S \approx 6$. These results indicate the persistance of spin clusters to temperatures significantly above the Curie temperature, even in nominally Heisenberg-like LSMO. Even more dramatic is the sharp downturn or knee in the LCMO inverse-susceptibility data and, to a lesser but still noticeable extent, in those for LPMO. This downturn, reported first by De Teresa et al. [26], moves to higher temperatures with increasing field. The scaling analysis shown in Fig. 2 include data only for $T / T_{C} \leq 1.06$; that is, at temperatures below 


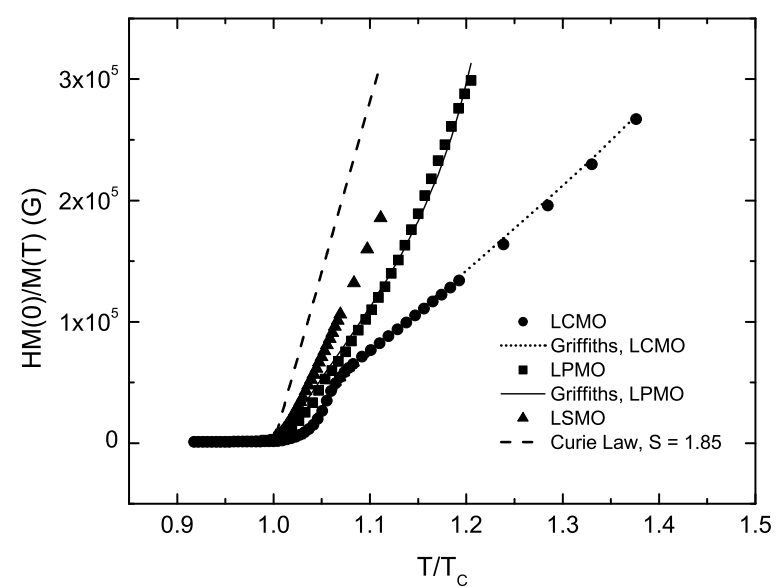

FIG. 4: Inverse susceptibility multiplied by the saturation magnetization. The dashed curve is the Curie-Weiss susceptility expected for $S=1.85$ and the critical temperature of the LSMO sample. The effective slope for LSMO corresponds to $S \simeq 3.5$, increasing to $S \simeq 6$ for LCMO. The curves through the data points are for the Griffiths model, as described in the text.

the downturn. We defer discussion of the other lines in Fig. 4 to the next section.

The anomalies in the magnetization are, of course, mirrored in the heat capacity data as functions of applied field. Figure 5a shows the data for LCMO and Fig. 5b, for LSMO at a succession of applied fields. The LCMO data shift to higher temperature while remaining relatively narrow while the LSMO data, as with other ferromagnets, broaden with little shift in peak position. As for the magnetization, the heat capacity data should collapse to a universal curve when scaled with a power of the magnetic field and plotted versus scaled temperature according to

$$
(C(H, T)-C(0, T)) H^{\alpha / \beta \delta}=f\left(\frac{t}{H^{1 / \beta \delta}}\right) .
$$

As we reported earlier,[27] neither the exponents that provide a scaling collapse of the magnetization data, nor any other set that we can identify, are able to satisfy the scaling conditions for LCMO. This is shown in Fig. 6a. However, the LSMO data, Fig. 6b, do fall on a single scaling curve using the values of $\beta$ and $\delta$ from the magnetization scaling, and $\alpha=-0.1$; the last differs slightly from a value consistent with $\beta$ and $\delta$. As the susceptibility data of Fig. 4 demonstrate, even LSMO does not exhibit single-spin behavior, so we must take the critical exponents to represent only effective values. 

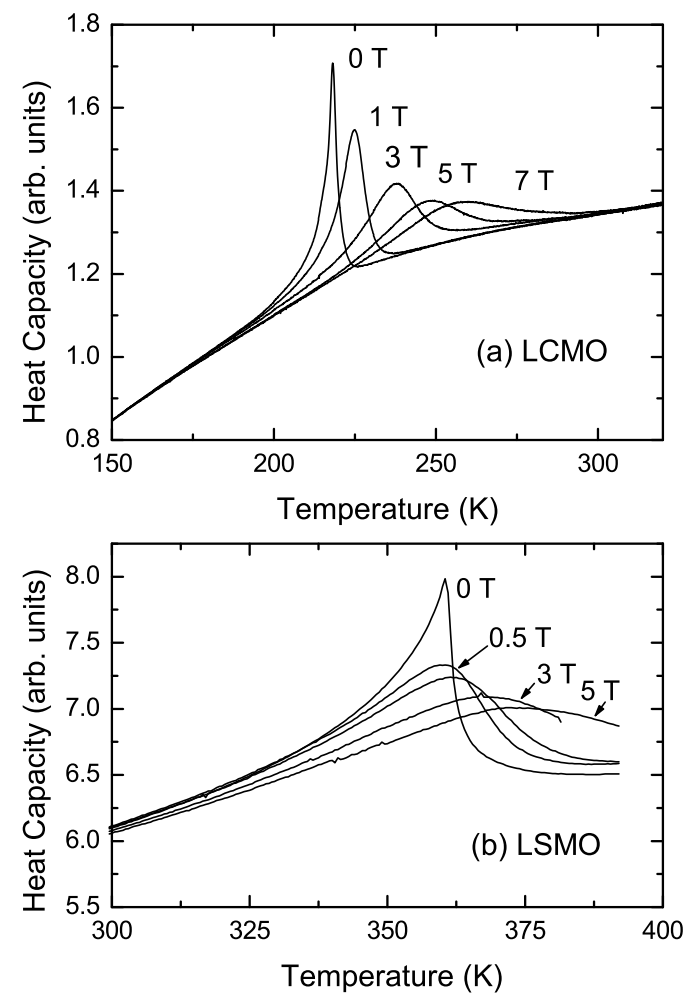

FIG. 5: Field dependence of the heat capacity of LCMO (a) and LSMO (b) at the listed fields. The curve for $B=1 \mathrm{~T}$ is not labeled in $\mathrm{b})$. Note the qualitatively different behavior of the two samples.

\section{GRIFFITHS PHASE ANALYSIS: SUSCEPTIBLITY}

In his pioneering 1969 paper, Griffiths [15] demonstrated that the magnetization of a randomly diluted ferromagnet above its percolation point is a non-analytic function of the field at all temperature below the pure-system Curie temperature. The argument was extended to alloys; i.e., for $0 \leq J_{2}<J_{1}$, by Fisch [16] and to any positive-definite (bounded) distribution of exchange interactions by Bray and Moore. [18] In the latter paper, the authors focused on the distribution $\rho(\lambda)$ of the eigenvalues $\lambda$ of the inverse susceptibility matrix. Above the critical temperature $T_{C}$ but below the highest achievable critical temperature $T_{G}$, all states with small values of $\lambda$ are localized; there are local regions of large susceptibility, but no long range order. Just at $T_{C}$, an extended state of infinite susceptibility $(\lambda=0)$ appears, signalling the sudden onset of long-range order. Subsequently, Bray [19] suggested 

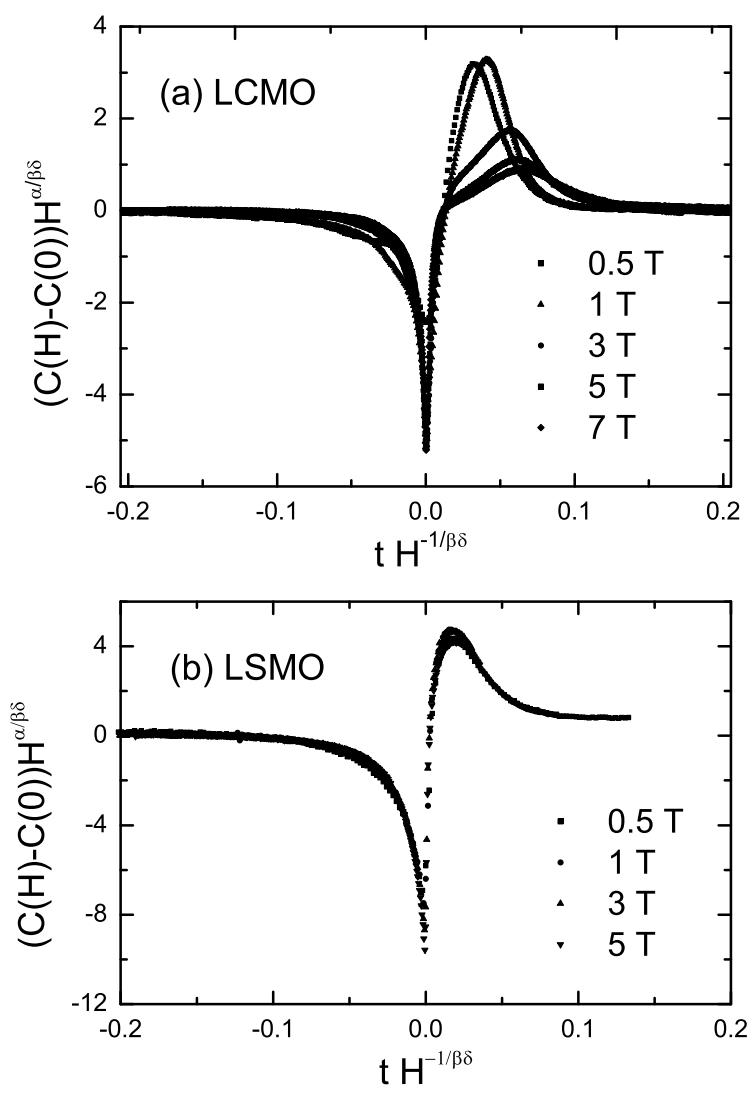

FIG. 6: The deviation of the heat capacity in magnetic field from its zero-field value, scaled by field and plotted versus scaled reduced temperature. No set of exponents can be found to collapse the LCMO data in a) to a single curve. By contrast, the same values of $\beta$ and $\delta$ used in Fig. 2, along with $\alpha=-0.1$ serve to collapse the LSMO data in b).

an explict form for this distribution,

$$
\rho(\lambda) \propto \lambda^{-x} \exp (-A(T) / \lambda)
$$

The power-law prefactor was not specified but Bray and Huifang later [28] considered a soluble model of the diluted Ising ferromagnet and verified Eq. (3) with $x=1 / 2$. The amplitude $A$ was argued to diverge as $\left(1-T / T_{G}\right)^{-2 \beta}$ at the pure, or Griffiths, temperature $T_{G}$ and to vanish as $\left(T / T_{C}-1\right)^{2(1-\beta)}$ at the actual Curie point. The exponent $\beta$ is the usual exponent for the system at its pure transition. This distribution peaks at $\lambda=A / x$ and vanishes at $\lambda=0$ for all temperatures above $T_{C}$. There is, therefore, a pile-up of small eigenvalues-large susceptibilities-as the Curie temperature is approached. Just at $T_{C}$ the distribution collapses into $\lambda=0$ causing the magnetization to jump to a large value in 


\begin{tabular}{|l|l|l|l|l|}
\hline & $a(\mathrm{~K})$ & $x$ & $T_{C}(\mathrm{~K})$ & $T_{G}(\mathrm{~K})$ \\
\hline LCMO & 5.0 & 0.53 & 224.8 & 376 \\
LPMO & 4.15 & 0.61 & 293.5 & 365 \\
\hline
\end{tabular}

TABLE II: Parameters used in Griffiths susceptibility calculation.

applied field-the hallmark of the Griffiths singularity.

We assert here that the transition temperatures evidenced in the sequence LSMO, LPMO and LCMO are a consequence of increased randomness due to the increased local bond bending in the vicinity of successively smaller dopant atoms. If so, then each sample is farther below the Griffiths temperature of an optimal system and will consequently exhibit a broader temperature range over which $A(T)$ varies between its zero at $T_{C}$ and its divergence at $T_{G}$. We calculate the average susceptibility from Eq. (3) according to

$$
\bar{\chi}=C \frac{\int_{0}^{T} \lambda^{-1} \rho(\lambda) d \lambda}{\int_{0}^{T} \rho(\lambda) d \lambda},
$$

where $C=n g^{2} \mu_{B}^{2} S(S+1) / 3 k_{B}$ is the Curie constant and the upper limit of the integral recognizes that the smallest susceptibility at any temperature is $C / T$ for spin $S$. The exponential amplitude is taken to be

$$
A(T)=a \frac{\left(T / T_{C}-1\right)^{2(1-\beta)}}{\left(1-T / T_{G}\right)^{2 \beta}},
$$

with $\beta=0.38$ and $a, T_{C}, T_{G}$ and $x$ varied to fit the susceptibility data. The down-turn in the inverse susceptibility curves sets the value of $T_{C}$ while the upward curvature is controled by $T_{G}$. There is considerable covarience of the amplitude $a$ and prefactor expononent $x$, so the values are subject to some uncertainty. We use the effective spin $S=1.85$ appropriate for $70 \% S=2$ and $30 \% S=1.5$. Because the downturn (if there is one) for LSMO is not discernable, we cannot get an unambiguous fit for those data. However, the solid curves for LPMO and LCMO are reliable, with the parameter values given in Table II.

Of considerable interest is the fact that the Griffiths temperatures that emerge from the fits are comparable and only slightly above the observed $T_{C}$ for LSMO. This indicates that LSMO lies very close to the optimal critical temperature and explains why it can be treated in the context of an ordinary Heisenberg ferromagnet, albeit with slightly modified critical exponents. 


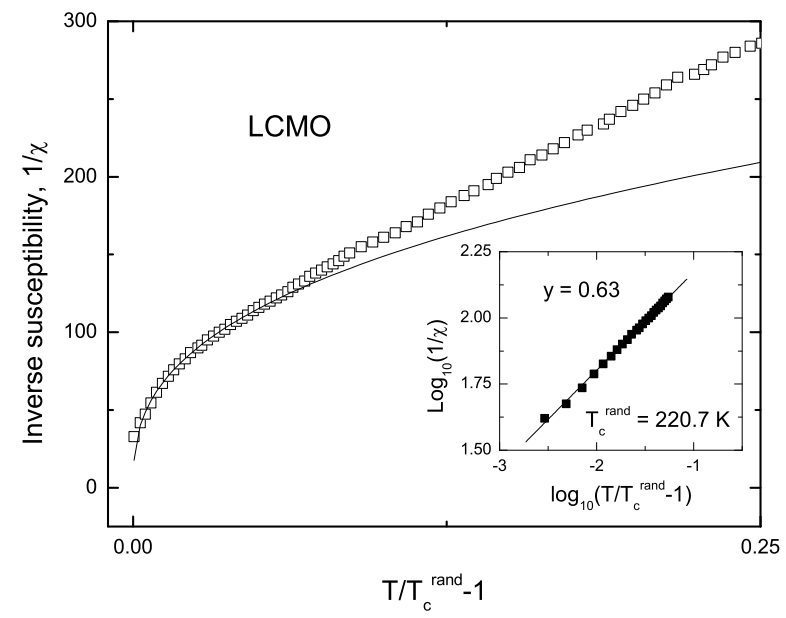

FIG. 7: Inverse susceptibility of LCMO at $500 \mathrm{Oe}$ as a function of $T / T_{c}$. The solid curve in the main figure and the logarithmic plot in the inset is a fit of the data to a power law with the result that $y=0.63$.

Note that the critical temperature obtained from the Griffiths fit is somewhat higher than that obtained from scaling or the heat capacity. This may reflect the suggestion made by Griffith in his original paper that the susceptibility would tend to diverge in advance of the onset of long-range order. To examine this, we focus on the downturn in the inverse susceptibility for LCMO. In recent work on f-electron compounds in which disorder has driven $T_{C}$ to $0 \mathrm{~K}$, Castro Neto, et al. [20] have argued that the susceptibility diverges as $T^{y-1}$ where $y \leq 1$ is related to the tunneling barrier for a cluster of $N$ aligned spins. In general, the relaxation rate of Griffiths clusters is also expected to be proportional to its inverse susceptibility [19], so similar arguments might hold here; i.e. $\chi^{-1} \propto\left(T / T_{C}-1\right)^{1-y}$. In Fig. 7 we plot the low-field susceptbility of the LCMO crystal as a function of $T / T_{C}$, with $T_{C}=220.7 \mathrm{~K}$ obtained by fitting the data to a power law. The random critical temperature is much closer to that indicated by the heat capacity peak, and is a more reliable measure of the tendency of the inverse susceptibility to vanish with an exponent $y=0.63$; that is, to approach $T_{C}$ with infinite slope. Though closer to the heat capacity peak $(218.2 \mathrm{~K}$ at this field) it appears to be somewhat above the temperature at which long-range order is established, as suggested by Griffiths. 


\section{GRIFFITHS PHASE ANALYSIS: HEAT CAPACITY}

In the classic Griffiths-phase model, exchange interactions are distributed randomly, but once distributed, are fixed. This is not the case for a double-exchange system in which the effective coupling between two Mn ions depends on the alignment of their respective core spins or, equivalently, the rate at which the outer $e_{g}$ electron hops between the two ions. As a consequence, as spins order locally, the spin clusters are also more metallic, and the combined effect is to reinforce and stabilize the formation of large, Griffiths clusters. In the presence of an applied magnetic field, these metallic, spin-aligned clusters form at higher temperatures, strongly affecting the thermodynamics of the transition and, of course, giving rise to the CMR effect itself.

The heat capacity associated with the Griffiths singularity was studied for the random spherical model by Rauh, [29] who found a jump singularity at $T_{C}$. We take a different approach here, using the Oguchi model [30] to calculate the magnetization and the associated short-range order parameter. In this approach, the interaction energy of a pair is calculated exactly using the double exchange energy

$$
E_{d e}\left(S_{t}\right)=-x t \frac{S_{t}+1 / 2}{2 S+1}-\overline{E_{d e}\left(S_{t}\right)}
$$

where the bar denotes an average over all possible values of the total spin $1 / 2 \leq S_{t} \leq 7 / 2$ of the two $S=3 / 2$ cores and the shared $e_{g}$ electron of the pair. The pair interacts with its $z-1$ neighbors through an effective magnetic field

$$
H_{\text {eff }}(H, T)=H+2(z-1) S\left\{c(H, t) J_{m e t}+[1-c(H, T)] J_{\text {ins }}\right\} m(H, T) .
$$

Here, $m(H, T)$ is the reduced magnetization to be calculated, $J_{m e t}$ is the exchange intereraction in metallic regions that have a concentration $c(H, T)$, and $J_{\text {ins }}$ is the exchange energy in non-metallic (but still conductive) regions. The insulating exchange energy can be extracted directly from the inverse susceptibility by extrapolating the linear region of Fig. 4 to obtain the Curie temperature $\Theta=202 \mathrm{~K}$, from which mean-field theory gives $J_{\text {ins }}=0.85 \mathrm{meV}$. We obtain $J_{\text {met }}$ from the spin-wave dispersion of manganites which is $D \approx 160 \mathrm{meV} \AA^{2}$ independent of concentration. The effective Heisenberg exchange interaction giving this spin-wave stiffness is $J_{m e t}=D / 2 S_{\text {eff }} a^{2}=1.56 \mathrm{meV}$; here $S_{\text {eff }}=1.85$ is the

average spin per manganese atom. The hopping energy giving the same spin-wave spectrum 


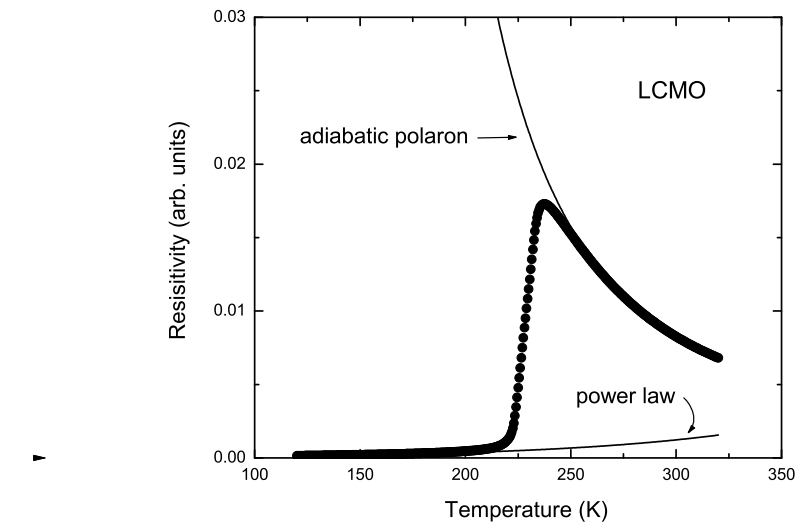

FIG. 8: Resistivity of the LCMO sample in a field of 1T. Superposed are the fits to the zero-field data at low tempratures to a power law and at high temperatures, to an adiabatic small polaron model.

is $t=D(2 S+1) / x a^{2}=140 \mathrm{meV}$. [31] Alternatively, the critical temperature from Monte Carlo simulations is $k_{B} T_{C} \approx 0.14 t$ [32], giving $t=134 \mathrm{meV}$.

The most important input into the model is the relative concentration of metallic bonds. We obtain this empirically from the resisitivity data as outlined in Fig.8. The zero-field data at low temperature are fit to the power law

$$
\rho_{l t}=\rho_{0}+a_{2} T^{2}+a_{5} T^{5}
$$

and the high-temperature data, to an adiabatic small-polaron contribution,

$$
\rho_{h t}=b T \exp \left(E_{p} / T\right)
$$

as done previously. [33] The metallic fraction is obtained by solving the generalized effective medium (GEM) expression [34] using the experimental resistivity $\rho_{\exp }$ and the extrapolated high and low temperature fits. The GEM approach guarantees that percolation occurs at a critical concentration $c_{c}$ which we set to the $3 \mathrm{D}$ value for spherical inclusions, namely $c_{c} \approx 1 / 6$. The equation to be solved for $c(H, T)$ is

$$
c(H, T) \frac{\rho_{\exp }^{1 / t}-\rho_{l t}^{1 / t}}{\rho_{\exp }^{1 / t}+A \rho_{l t}^{1 / t}}+[1-c(H, T)] \frac{\rho_{\exp }^{1 / t}-\rho_{h t}^{1 / t}}{\rho_{\exp }^{1 / t}+A \rho_{h t}^{1 / t}}=0,
$$

where $A=\left(1-c_{c}\right) / c_{c}$ and the percolation exponent is set to $t=2$. Several resistivity curves and the resulting concentrations are shown in Fig. 9. 


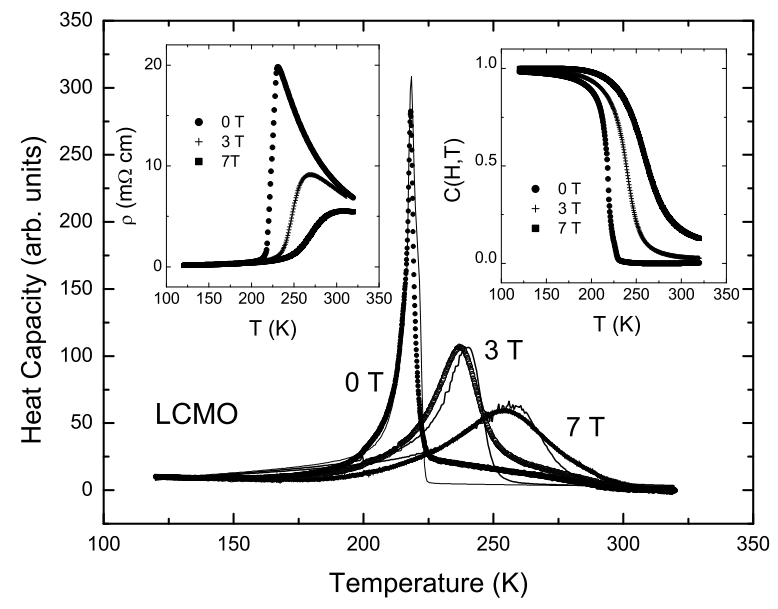

FIG. 9: Heat capacity curves calculated from the Oguchi model at several fields. The metallic concentrations extracted from the resistivity curves (left inset) are shown in the right inset. The only other input is the overall amplitude of zero-field curve.

We proceed by calculating the magnetization self-consistently in the context of the Oguchi model; that is, we solve

$$
m(H, T)=\frac{1}{Z} \sum_{S_{t}=1 / 2}^{7 / 2} \sum_{p=S_{t}}^{S_{t}} p \exp \left(\frac{-E_{d e}+p g \mu_{B} H_{e f f}(H, T)}{k_{B} T}\right),
$$

where $Z$ is the partition function (same sum without the factor $p$ ). Once $m(H, T)$ is known, we compute the energy density by averaging $E_{d e}\left(S_{t}\right)$ at each field/temperature point using the Boltzmann factors that have been calculated self-consistently, and differentiate numerically to obtain the heat capacity. The amplitude is chosen to fit the zero-field data and kept constant for other fields. The results are shown in Fig. 9. The width, amplitude, and shift in temperature of curves at successive fields agrees extremely well with the data. In each case, the experimental peaks are broader on the high-temperature side of the curve, indicating that the Oguchi calculation underestimates the persistence of shortrange order to higher temperatures. Nonetheless, the curves show quite clearly that the metallic concentrations extracted from the GEM analysis are able to predict the unusual critical behavior of LCMO.

The final question in this analysis is whether the Oguchi model described here actually reproduces Griffiths-like behavior at low fields. The magnetization has been calculated at the same fields as the data in Fig.4, using the zero-field value $c(0, T)$ extracted from the 


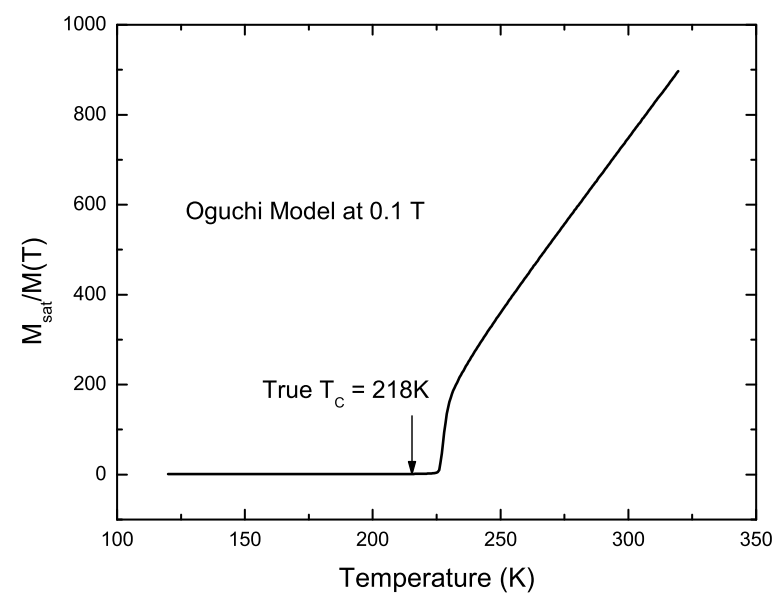

FIG. 10: Inverse susceptibility at low field calculated using the Oguchi model. The sharp downturn at a temperature above the peak in the heat capacity peak mirrors the behavior of the experimental data.

resistivity. The result is shown in Fig.10. Note that the down-turn in advance of the heat capacity peak is similar to the experimental data. The Oguchi approach does not capture the persistence of spin clusters to the Griffiths temperature.

\section{CONCLUSIONS}

The nature of the ferromagnetic-paramagnetic transition in these materials has been subject to considerable discussion. Most recently, Kim, et al.[35]argued for a tricritical point just above $x=0.33$ in $\mathrm{La}_{1-x} \mathrm{Ca}_{x} \mathrm{MnO}_{3}$. They do not identify the two phase lines that emanate from the tricritical point. Further, examination of the $x=0.33$ data shows that the susceptibility at $290 \mathrm{~K}$ corresponds to a spin $S=3.5$, rather than the $S \leq 2$ expected. Consequently, even $30 \mathrm{~K}$ above the transition there is evidence for clustering, a signature of the Griffiths behavior we propose here. Apart from the critical behavior, various explanations for the CMR phenomenon drawn upon elements of the Griffiths-phase approach-mixed phases and phase separation, percolation, slow dynamics-but have not connected them into a coherent picture. In particular, the dramatic changes in behavior that accompany subtle changes the size and concentration of dopant atoms have not been adequately treated. We have attempted here to demonstrate that the intrinsic randomness introduced by substituting ions that differ in size (and of course valence) from the usual A- 
site atom drive the system from its optimal doping and ionic size at $\mathrm{Sr}_{3 / 8}$ to the strong CMR regime as $\mathrm{Sr}$ is changed to $\mathrm{Pb}$ and finally $\mathrm{Ca}$. Remarkably, the transition to the magnetic phase remains second-order like, by which we mean that the properties are fully reversible and, with the exception of the heat capacity, can be treated by the usual ferromagnetic scaling equations, albeit with non-universal (even bizarre) values for the critical exponents.

Outside the "critical" regime, there is ample evidence in our data, and in a wealth of further data in the literature, to demonstrate coexistence of more or less metallic and more or less insulating regions over a wide temperature range both above and below the Curie temperature. We have shown that the clusters evolve as the temperature is reduced toward $T_{C}$ in a manner consistent with the theoretical ideas of Bray and Moore [18] and Bray [19]. In essence, the transition is not primarily a question of connectedness and the evolution of a tenuous infinite cluster, but rather more a homogeneous nucleation problem in which the most-probable cluster size grows as the temperature is reduced until they become effectively space-filling, providing an abrupt onset of nearly complete long-range order.

The situation in the manganites differs significantly from straightforward Griffiths phase precisely because Griffiths clusters are more metallic and therefore more ferromagnetic than the surrounding matrix. the CMR effect thus reinforces cluster formation: local spin ordering increases the mobility of electrons, which then increases local exchange interactions via double exchange, which in turn feeds back to lock local spin ordering. We have attempted to deal with this effect phenomenologically by determining the fraction of metallic, high-susceptibility clusters from the field and temperature dependent resistivity using a generalized effective medium approach. Knowing that fraction, we compute an effective magnetic field acting on each pair of double-exchange coupled spins and from that, determine the magnetization and energy density. We demonstrate that this approach accurately tracks the height and temperature of the peak in the heat capacity and, to a significant extent, its width. We regard the unusual behavior of the heat capacity in magnetic field, along with the strongly non-Curie-Weiss behavior of the susceptiblity to be hallmarks of the CMR effect, as important in understanding it as the more dramatic changes in transport property.

Our analysis of the CMR transition in terms of Griffiths-phase ideas provides an understanding of the evolution of behavior from LSMO, whose Curie point is near the Griffiths temperature, to LCMO, which exhibits Griffiths phase and magnetotransport signatures. 
However, the interplay of local order and enhanced double exchange requires empirical input and remains, therefore, unsatisfactory. We still need to understand the mechanism by which Griffiths clusters order in a polaronic, double-exhange magnet, and how that process assists in stabilizing large clusters. It is our hope that this paper has helped to delineate the problems that remain.

\section{ACKNOWLEDGEMENTS}

This material is based upon work supported by the U.S. Department of Energy, Division of Materials Sciences under Award No. DEFG02-91ER45439, through the Frederick Seitz Materials Research Laboratory at the University of Illinois at Urbana-Champaign. We are

especially grateful for the crystals provided by T. Okuda, Y. Tomioka, and A. Asamitsu in the group of Prof. Y. Tokura. We have also benefited from discussions with Marcelo Jaime, Yuli Lyanda-Geller, and Paul Goldbart.

[1] M. B. Salamon and M. Jaime, Rev. Mod. Phys. 73, 583 (2001).

[2] H. Hwang, S.-W. Cheong, P. Radaelli, M. Marezio, and B. Batlogg, Phys. Rev. Lett. 75, 914 (1995).

[3] L. Rodriguez-Martinez and J. Attfield, Phys. Rev. B 54, R15622 (1996).

[4] K. Ghosh, C. Lobb, R. Greene, S. Karabashev, D. Shulyatev, A. Arsenov, and Y. Mukovskii, Phys. Rev. Lett. 81, 4740 (1998).

[5] C. Zener, Phys. Rev. 2, 403 (1951).

[6] A. Urushibara, Y. Moritomo, T. Arima, A. Asamitsu, G. Kido, and Y. Tokura, Phys. Rev. B 51, $14103(1995)$.

[7] A. Millis, P. Littlewood, and B. I. Shraiman, Phys. Rev. Lett. 74, 5144 (1995).

[8] A. J. Millis, R. Mueller, and B. I. Shraiman, Phys. Rev. B 54, 5405 (1996).

[9] M. Jaime, M. B. Salamon, M. Rubinstein, R. E. Trece, J. Horwitz, and D. B. Chrisey, Phys. Rev. B 54, 11914 (1996).

[10] J. L. Garcia-Munoz, J. Fontcuberta, M. Suaaidi, and X. Obradors, J. Phys.: Cond. Matt. 8, L787 (1996). 
[11] V. Podzorov, M. Uehara, M. E. Gershenson, T. Y. Koo, and S.-W. Cheong, Phys. Rev. B 61, $3784(2000)$.

[12] R. Merithew, M. Weissman, F. Hess, P. Spradling, E. Nowak, J. ODonnell, J. Eckstein, Y. Tokura, and Y. Tomioka, Phys. Rev. Lett. 84, 3442 (2000).

[13] R. Heffner, J. Sonier, D. MacLaughlin, G. Nieuwenhuys, G. Ehlers, F. Mezei, S. Cheong, J. Gardner, and H. Roder, Phys. Rev. Lett. 85, 3285 (2000).

[14] E. Dagotto, T. Hotta, and A. Moreo, Phys. Repts 344, 1 (2001).

[15] R. B. Griffiths, Phys. Rev. Lett. 23, 17 (1969).

[16] R. Fisch, Phys. Rev. B 24, 5410 (1981).

[17] V. S. Dotsenko, J. Phys. A:Math Gen 32, 2949 (1999).

[18] A. J. Bray and M. A. Moore, J. Phys. C:Solid State Phys. 15, L765 (1982).

[19] A. J. Bray, Phys. Rev. Lett. 59, 586 (1987).

[20] A. H. Castro Neto, G. Castilla, and B. A. Jones, Phys. Rev. Lett. 81, 3531 (1998).

[21] A. H. C. Neto and B. A. Jones, Phys. Rev. B 62, 14975 (2000).

[22] M. B. Salamon, P. Lin, and S. H. Chun, Phys. Rev. Lett. 88, 197203 (2002).

[23] T. Okuda, Y. Tomioka, A. Asamitsu, and Y. Tokura, Phys. Rev. B 61, 8009 (2000).

[24] M. Jaime, P. Lin, M. B. Salamon, and P. D. Han, Phys. Rev. B 58, R5901 (1998).

[25] Y. Kraftmakher, Phys. Rept. 356, 1 (2002).

[26] J. M. De Teresa, M. R. Ibarra, P. A. Algarabel, C. Ritter, C. Marquina, J. Blasco, J. Garcia, A. del Moral, and Z. Arnold, Nature 386, 256 (1997).

[27] P. Lin, S. H. Chun, M. B. Salamon, Y. Tomioka, and Y. Tokura, J. Appl. Phys. 87, 5825 (2000).

[28] A. J. Bray and D. Huifang, Phys. Rev. B 40, 6980 (1989).

[29] A. Rauh, Phys. Rev. B 14, 1997 (1976).

[30] J. S. Smart, Effective Field Theories of Magnetism (W. B. Saunders, Philiadelphia, 1966).

[31] N. Ohata, J. Phys. Soc. Jpn 34, 343 (1973).

[32] M. J. Calderon and L. Brey, Phys. Rev. B 58, 3286 (1998).

[33] M. Jaime, P. Lin, S. H. Chun, M. B. Salamon, P. Dorsey, and M. Rubinstein, Phys. Rev. B 60, 1028 (1999).

[34] G. Hurvits, R. Rosenbaum, and D. S. McLachlan, J. Appl. Phys. 73, 7441 (1993).

[35] D. Kim, B. Revaz, B. L. Zink, F. Hellman, J. J. Rhyne, and J. F. Mitchell, Phys. Rev. Lett. 
89, 227202 (2002). 